\title{
STUDIES OF THE LARGE MAGELLANIC CLOUD USING OPTICAL INTERSTELLAR EMISSION LINES
}

\author{
J. MEABURN \\ Department of Astronomy \\ The University \\ Manchester M13 9PL \\ England
}

ABSTRACT. Optical observations have been made of the halo of 30 Doradus, in the vicinity of SN 1987A and of giant shells in the LMC.

\section{Introduction}

Spatially resolved observations of profiles of optical emission lines combined with deep $\mathrm{H} \alpha$ photographs have revealed a complex array of kinematical and morphological phenomena along many sight-lines through the Large Magellanic Cloud (LMC). Localised motions of several origins, invariably associated with bursts of star formation in the vicinity of giant interstellar shells, are superimposed on the pre-existing motions of the giant molecular clouds which constitute the spiral features of the young LMC (McGee and Milton 1966, Feitzinger and Weiss 1979). The kinematics and morphology of some particularly significant regions will be illustrated by comparing positional - velocity ( $p-v)$ arrays of emission line profiles with deep images. These profiles were obtained principally with the Manchester echelle spectrometer (Meaburn et al. 1984).

\section{Halo of 30 Doradus}

The only truly massive HII complex in the LMC, 30 Dor, occupies a unique position as the nucleus of the spiral features of the youngest phenomena (Schmidt-Kaler and Feitzinger 1976). Its dense ionized core is energised by the $O B$ association around R136. Filamentary ionized shells whose diameters $(30-100 \mathrm{pc})$ increase with distance from the core surround separate $\mathrm{OB}$ associations distributed throughout the halo. These giant shells culminate in the two supergiant shells that can be seen in Figure 1 (Meabum 1979 and 1980). The lines along which spatially resolved profiles of $\mathrm{H} \alpha$ and [OIII] emission lines were obtained (Meaburn 1981, 1984 and 1988) are shown against a sketch of the ionized filaments and star clusters of 30 Dor in Figure 2.

It is important to realise that the application of different analytical techniques to the same longslit spectrum reveals distinctly separate types of kinematical behaviour. For example, Gaussian fitting of complex profiles is most useful for tracing separate velocity components along a $\mathrm{p}-\mathrm{v}$ array. Contouring is poor for this purpose but permits the display of high-velocity spikes etc. where Gaussian fitting has no application.

The positions of components with separate radial velocities, after simulation of the line profiles by multiple Gaussians, are depicted in Figure 3a for the NS line in Figure 2 (Meaburn 1981) and in Figure $3 \mathrm{~b}$ for one of the EW lines in Figure 2 (Meaburn 1984). Only components which appear as 


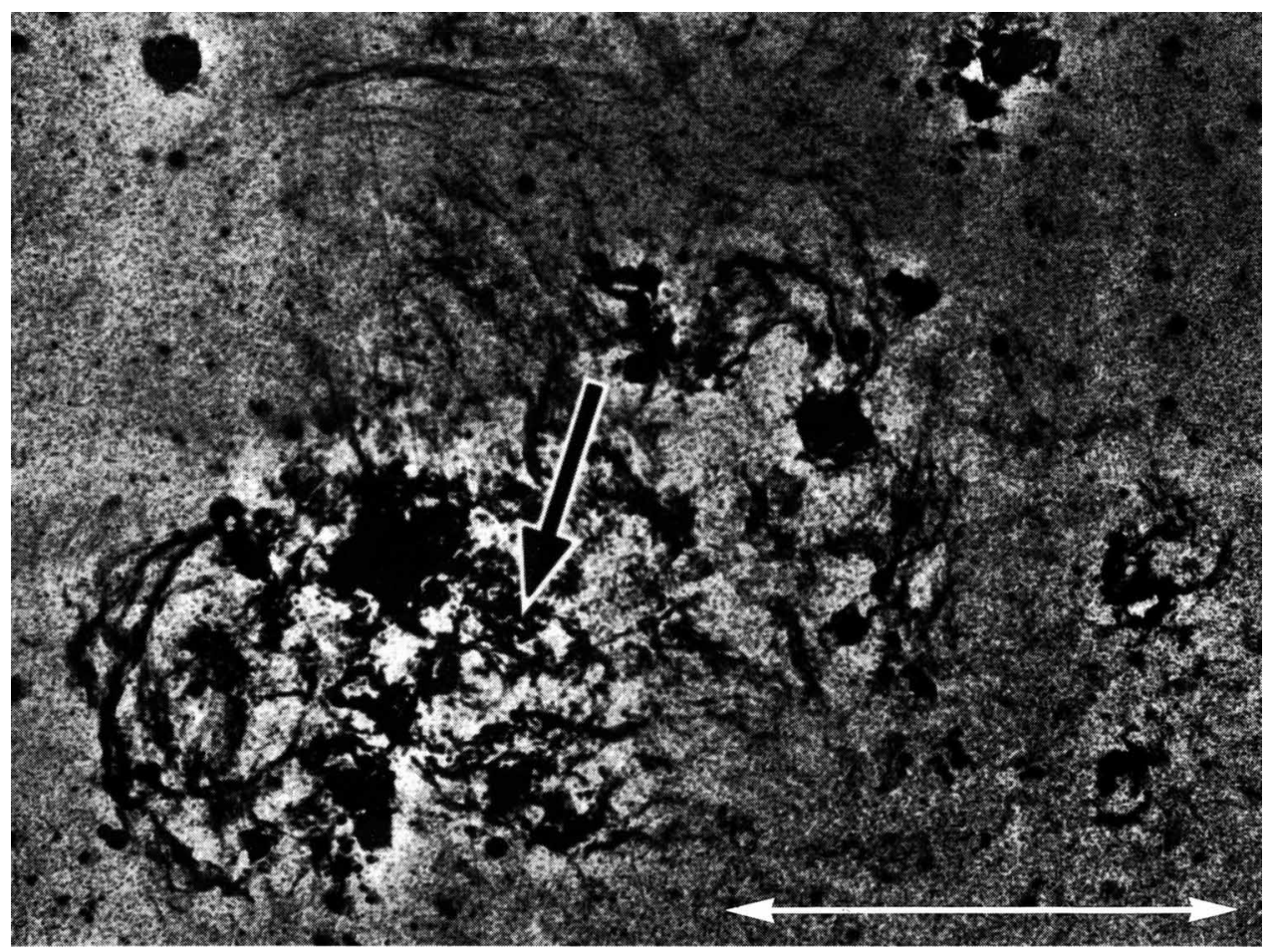

Figure 1. A deep H $\alpha$ photograph of the vicinity of 30 Dor. SN 1987A is arrowed. The white line is 1 Kpc long.

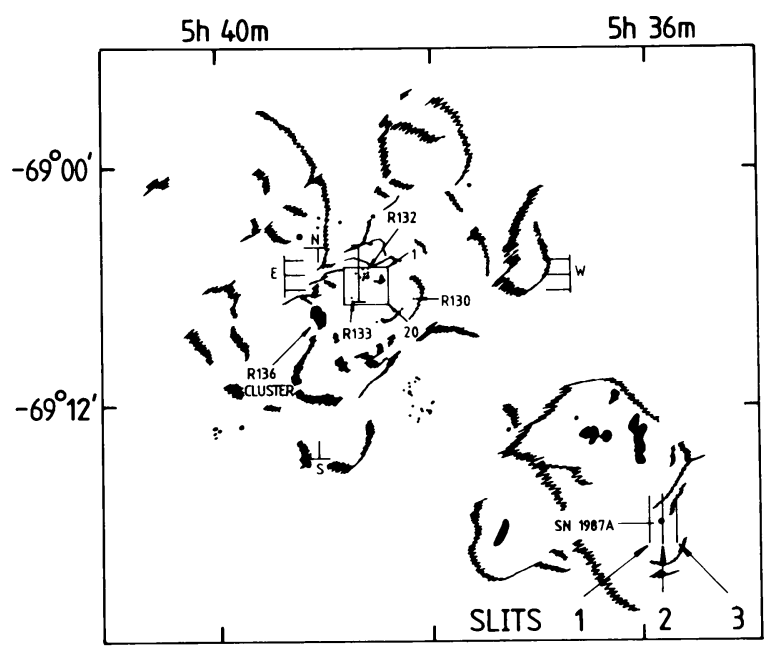

Figure 2. Paths along which optical line profiles have been obtained over 30 Dor and near SN 1987A. 
distinct maxima in the profiles are included to eliminate any possibility of over-interpretation. The extents of non-Gaussian wings to profiles are shown as arrowed lines in Figure $3 \mathrm{~b}$. High velocity ridges are contained within dashed lines. The nature of one of these is better displayed in the contour map in Figure 4a for the p-v array over the cluster containing R132 (Figure 2).

The existence of a sheet of ionized gas in the halo of 30 Dor with a high radial velocity $(\approx-200$ $\mathrm{km} / \mathrm{s}$ ) with respect to the systemic (Figure $3 \mathrm{~b}$ ) is also illustrated in Figure $4 \mathrm{~b}$, where an extensive feature with $\mathrm{V}_{\mathrm{HEL}}=90-130 \mathrm{~km} / \mathrm{s}$ is present along the southern slit lengths of the box of slit positions around R132 in Figure 2 (also see Chu and Kennicutt, 1990).

Some sense can be made out of this confusion of features in the p-v arrays. Firstly, bright, extensive velocity components are present which are coincident with those at $V_{H E L} \approx 246,265$, 277 and $296 \mathrm{~km} / \mathrm{s}$ in the HI $21 \mathrm{~cm}$ profiles over this region. These must then have been produced as the primeval molecular clouds became photoionized by the Lyman flux from the newly formed stars.

Continuous features abound in the $\mathrm{p}-\mathrm{v}$ arrays which systematically diverge in radial velocity by $30-50 \mathrm{~km} / \mathrm{s}$ over distances of $25-50 \mathrm{pc}$ (for diameters of $50-100 \mathrm{pc}$ ). Radial expansions of the approximately spherical, giant shells in the halo of 30 Dor are implied. If the extreme sheet with $\mathrm{V}_{\mathrm{HEL}} \approx 100 \mathrm{~km} / \mathrm{s}$ (Figures $3 \mathrm{~b}$ and $4 \mathrm{~b}$ ) has this origin then an expansion velocity for this giant shell would be $200 \mathrm{~km} / \mathrm{s}$. Alternatively, this extensive, high-speed feature may represent a centrallydriven outflow from the vicinity of R136 (Meaburn 1981).

Every sight-line through the halo of 30 Dor then intersects several giant shells each with a different expansion velocity. Any explanation of these halo giant shells must also apply to those giant shells in the LMC which surround isolated OB associations (see the discussion in Section 4). The supergiant shells near 30 Dor (Figure 1) may be spherical but those well away are most likely toroidal. In all cases, stochastic star formation in their expanding perimeters is their most likely origin.

The most dramatic features in the p-v arrays over the halo of 30 Dor are the $\approx 15 \mathrm{pc}$ diameter regions emitting complex profiles which extend out to $\pm 250 \mathrm{~km} / \mathrm{s}$ (eg. Figures $3 \mathrm{a}$ and $4 \mathrm{a}$ ). That around the R132 cluster (Figures 2 and 4a) has all the characteristics of a high-speed expanding shell. Bursts of stellar wind from enclosid O-type or WR stars could produce energy-conserving bubbles such as these in $\leq 10^{4}$ yrs (Dyson and de Vries 1972). However, these features are more likely manifestations of the remnants of Type II supemova explosions of similar ages. In the limited area of 30 Dor sampled here (say 10\% - see Figure 2) there are $\approx 4$ of these $15 \mathrm{pc}$ diameter high-speed regions, in which case $\approx 40$ could be expected, in the whole halo of 30 Dor, to give an average time between explosions $\tau \leq 250 \mathrm{yr}$ if the supernova explanation applies. The halo of 30 Dor may then be a region of exceptionally high supernova activity. (Mathewson et al. 1983 give $\tau$ $\approx 275 \mathrm{yr}$ for the rest of the LMC.)

\section{SN 1987A}

Extensive ( $\geq 30 \mathrm{pc}$ across) clouds of ionized gas with distinctly separate radial velocities of $\mathrm{V}_{\mathrm{HEL}}=$ $255,280,300$ and $318 \mathrm{~km} / \mathrm{s}$ are revealed (Meaburn 1990) in the p-v arrays of [OIII] profiles in the vicinity of SN 1987A (slits 1-3 in Figure 2 and see Figure 5). Those at 255 and $280 \mathrm{~km} / \mathrm{s}$ closely match velocity components in the interstellar absorption line profiles found in the light of SN 1987A (see Pettini 1988 and Savage et al. 1989 for a summary of many authors' work), and must consequently be from sheets of neutral and ionized interstellar gas on the nearside of the explosion (i.e. the nearsides of giant shells). The 300 and $318 \mathrm{~km} / \mathrm{s}$ clouds are likely to be on the far side. 


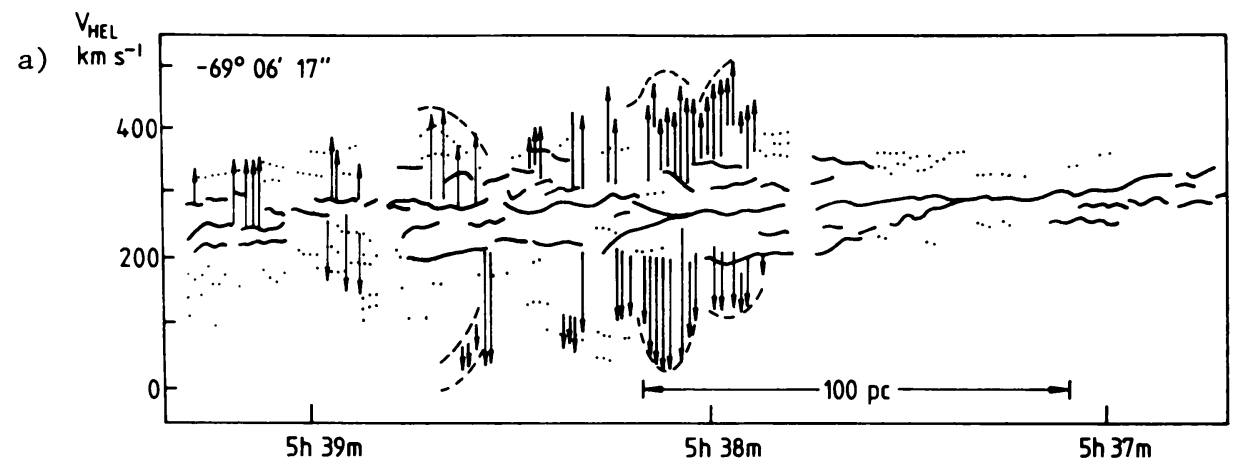

b)

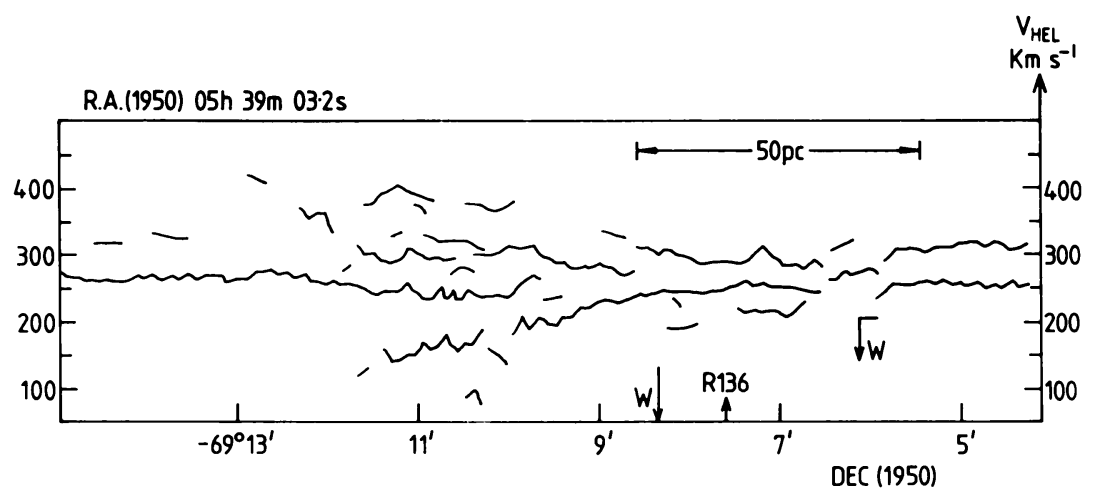

Figure 3. Separate velocity components along the a) EW and b) NS lines marked in Fig. 2 are traced by heavy solid lines for major features. Arrowed lines imply wings. High speed shells are contained within the dashed lines.

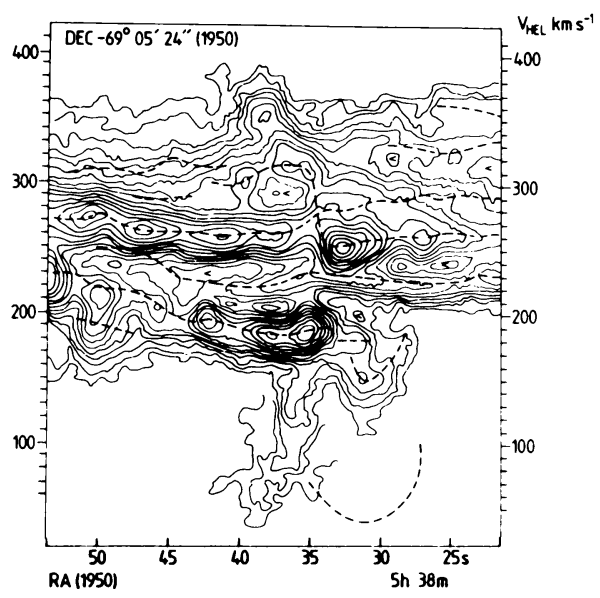

Figure 4a. Contours of the p-v array of [OIII] profiles from the EW line over R132 in Fig. 2. 


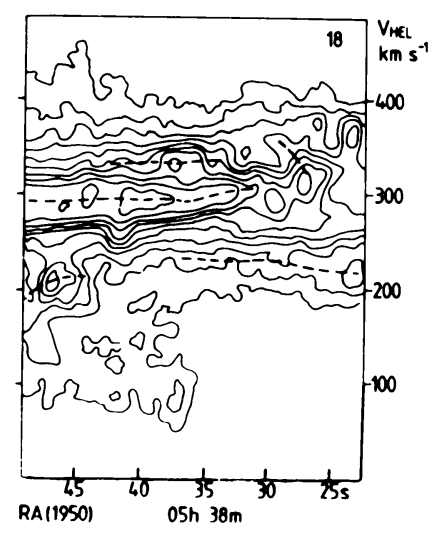

Figure 4b. As for a) but from the southern part of the box around R132 also shown in Fig. 2.
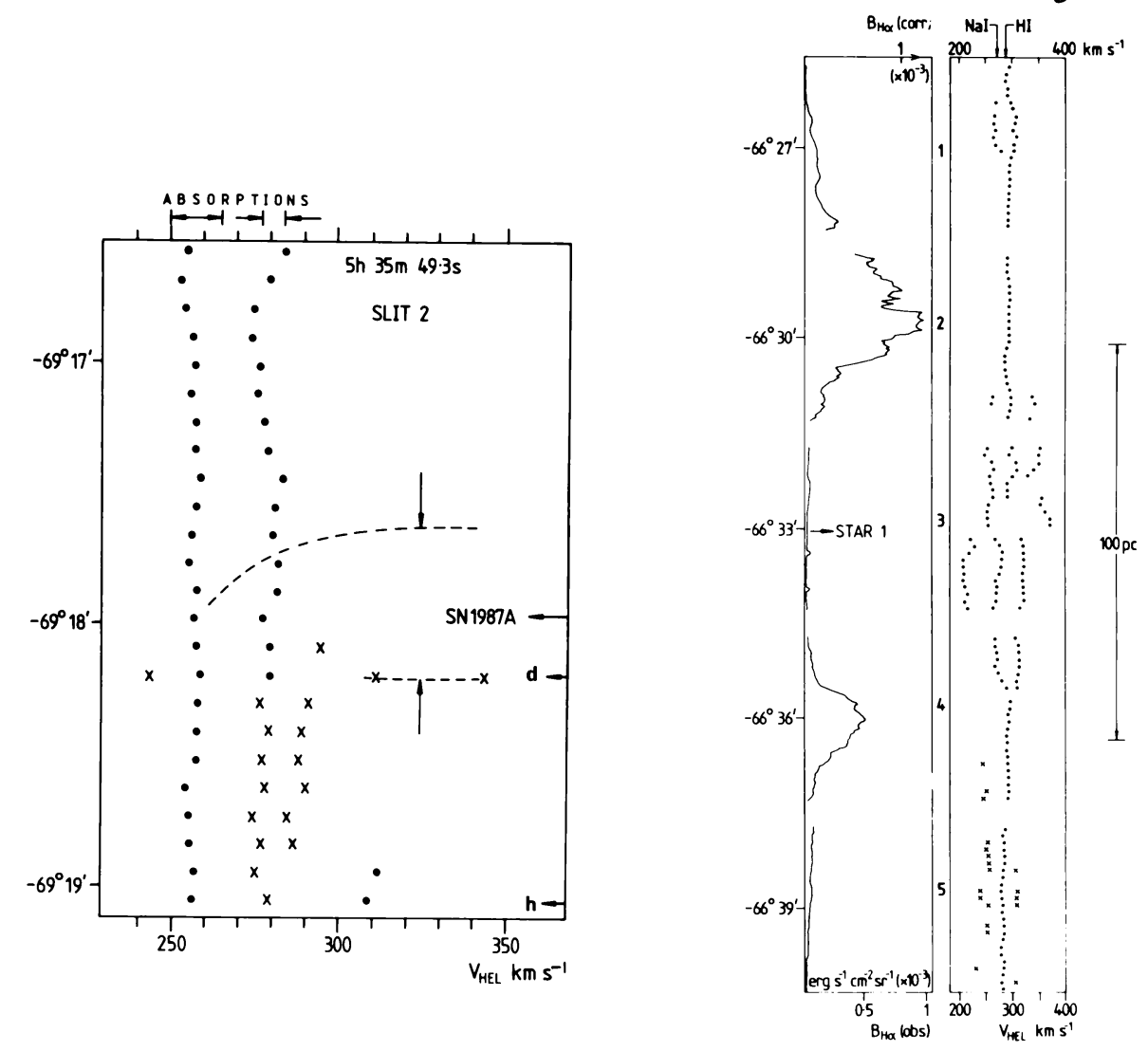

Figure 5. Velocity components in [OIII] profiles along slit (2) in Fig. 2 over SN 1987A. The dashed lines mark ridges.

Figure 6. Velocity components along a line through the giant shell N11 (DEM 34). 
In the p-v array for slit 2 in Figure 2, which is the closest to SN 1987A, two ridges project $\approx 100 \mathrm{~km} / \mathrm{s}$ towards positive radial velocities on either side of the supernova (shown as dashed curves in Figure 5). Although higher signal-to-noise ratios are required to map these features completely, an elongated cavity $\approx 8 \mathrm{pc}$ across could be present, with outflowing walls, similar to one lobe of many galactic bi-polar nebulae.

It is proposed that the precursor star, in its red giant phase, emitted a slow, dense wind which formed a disc. The later hypersonic, supergiant wind could then have driven elongated cavities perpendicular to the plane of the disc. The remnant is now expanding into this most likely bi-polar structure.

\section{Giant shells}

There are three sub-groups in the classification of giant (20 - $350 \mathrm{pc}$ diameter) LMC shells (see Meaburn et al. 1989 and see refs. therein for many authors' optical and other observations). Evolved supernova remnants appear as isolated, fine filamentary shells. Filamentary shells also surround OB associations, and shells with more amorphous filaments surround old associations with younger associations in their perimeters.

Stellar winds from OB and WR stars could combine with a modest number of successive supernova explosions in the central OB associations to pressurise the expansions of the latter two types of giant shell. Alternatively, supernova explosions could dominate all other sources if these events are sufficiently frequent.

An attempt has been made (Meaburn et al 1989) in recent observations of the amorphous, 350 pc diameter LMC giant shell, N11 (DEM 34) to investigate these possibilities quantitatively. Complex radial expansion of $\approx 80 \mathrm{~km} / \mathrm{s}$ (see Figure 6) is occurring in the tenuous ionized gas over the central and oldest OB association, LH9, which emits Lyman photons at the same rate as would $7 \pm 4$ supergiant 05 type stars. The expanding ionized gas has an observed kinetic energy $\mathrm{KE}$ $(\mathrm{Obs}) \approx 2.3 \times 10^{50} \mathrm{erg}$ and momentum $\mathrm{P}(\mathrm{Obs}) \approx 10^{44} \mathrm{~g} \mathrm{~cm} / \mathrm{s}$. If the energy conversion efficiency is defined as $\varepsilon_{\mathrm{E}}=\mathrm{KE}(\mathrm{Obs}) / \mathrm{KE}$ (In) and momentum efficiency as $\varepsilon_{\mathrm{P}}=\mathrm{P}(\mathrm{Obs}) / \mathrm{P}(\mathrm{In})$ then for energy conserving, pressure driven bubbles $\varepsilon_{\mathrm{E}}=0.2$ and $\varepsilon_{\mathrm{P}} \gg 1$, and for momentum conserving shells (snowploughs) $\varepsilon_{\mathrm{P}}<<1$ and $\varepsilon_{\mathrm{P}} \approx 1$.

If Type II LMC explosions occur predominantly in OB associations and if their probability of occurence is naively taken to be proportional to the total Lyman flux from each association, then around $160 \pm 80$ explosions are expected in the lifetime ( $\left.<10^{7} \mathrm{yr}\right)$ of $\mathrm{LH} 9$ to drive the expansion of N11.

With this number, then $\varepsilon_{\mathrm{E}} \approx 0.001$ and $\varepsilon_{\mathrm{P}} \approx 0.3$, which suggests that the motions are those of momentum conserving shells with winds playing little part in their generation. However, a better method of estimating the rate of supernova explosions in OB associations is needed before any certain conclusions are drawn. This may arise from X-ray observations similar to those of Chu and Low (1990). They show remnants in collision with the inner surfaces of giant LMC shells.

\section{References}

Chu, Y. and Kenniculi, R.C. (1990), this volume.

Chu, Y. and Low, M.M. (1990), Astrophys. J. in press.

Dyson, J.E. and de Vries, J. (1972), Astron. Astrophys. 20, 223. 
Feitzinger, J.V. and Weiss, G. (1979), Astron. Astrophys. Suppl. 37, 575.

Mathewson, D.S., Ford, V.L., Dopita, M.A., Tuohy, I.R., Long, K.S. and Helfand, D.J. (1983), Astrophys. J. Suppl. 51, 345.

McGee, R.X. and Milton, J.A. (1966), Australian J. Phys. 19, 343.

Meaburn, J. (1979), Astron. Astrophys. 75, 127.

Meaburn, J. (1980), Mon. Not. R. Astron. Soc. 192, 365.

Meaburn, J. (1981), Mon. Not. R. Astron. Soc. 196, 19P.

Meaburn, J. (1984), Mon. Not. R. Astron. Soc. 211, 521.

Meaburn, J. (1988), Mon. Not. R. Astron. Soc. 235, 375.

Meaburn, J. (1990), Mon. Not. R. Astron. Soc. 244, 551.

Meaburn, J., Blundell, B., Carling, R., Gregory, D.F., Keir, D. and Wynne C.G. (1984), Mon. Not. R. Astron. Soc. 210, 463.

Meaburn, J., Solomos, N., Laspias, V. and Goudis, C.D. (1989), Astron. Astrophys. 225, 497. Pettini, M. (1988), Proc. Astr. Soc. Australia 7, 527.

Savage, B.D., Jenkins, E.B., Joseph, C.L. and de Boer, K.S. (1989), Astrophys. J. 345, 393.

Schmidt-Kaler, Th. and Feitzinger, J.V. (1976), Astrophys. Space Sci. 41, 357. 